Europhysics Letters

PREPRINT

\title{
Dynamic ultrametricity in finite dimensional spin glasses
}

\author{
Daniel A. Stariolo $(*)$ \\ Instituto de Física, Universidade Federal do Rio Grande do Sul \\ CP 15051, 91501-970 Porto Alegre, Brazil \\ PACS. 75.10.Nr - Spin-glass and other random models. \\ PACS. 75.40.Mg - Numerical simulation studies. \\ PACS. 05.70.Ln - Nonequilibrium and irreversible thermodynamics.
}

\begin{abstract}
We show results of simulations of a weakly driven four dimensional EdwardsAnderson spin glass, which present clear signatures of dynamical ultrametricity at low temperatures. The presence of a hierarchical organization of time scales is evident from the appearance of a triangular relation between correlations at three characterisitc long times in the asymptotic limit of small drive, and also in the scaling form of stationary correlations in this regime. Recent results in the three dimensional case have been inconclusive showing the importance of dimensionality in tackling this delicate problem.
\end{abstract}

Introduction. - Ultrametricity between points or states in the phase space of complex systems is a remarkable property which signals a hierarchical organization of the states [1, 2]. In an $\mathrm{N}$ dimensional space, a square distance between two states $\alpha$ and $\beta$ with coordinates $s_{i}^{\alpha}= \pm 1, i=1 \ldots N$, can be defined as:

$$
\begin{aligned}
\left(d^{\alpha \beta}\right)^{2} & =\frac{1}{2 N} \sum_{i=1}^{N}\left(s_{i}^{\alpha}-s_{i}^{\beta}\right)^{2} \\
& =1-q^{\alpha \beta}
\end{aligned}
$$

where $q^{\alpha \beta}=(1 / N) \sum_{i} s_{i}^{\alpha} s_{i}^{\beta}$ is the overlap between the states. If $\alpha, \beta$ and $\gamma$ are three states, then ultrametricity means that:

$$
d^{\alpha \gamma} \leq \max \left\{d^{\alpha \beta}, d^{\beta \gamma}\right\}
$$

or equivalently:

$$
q^{\alpha \gamma} \geq \min \left\{q^{\alpha \beta}, q^{\beta \gamma}\right\}
$$

Ultrametricity is a distinctive feature of the phase space of the Sherrington-Kirkpatrick spin glass [2]. The search for ultrametricity in more realistic short range models has been acomplished by searching for ground states of small systems [3] or thermalized states at not too low temperatures 4 .

$\left({ }^{*}\right)$ Regular Associate of the Abdus Salam International Center for Theoretical Physics, Strada Costiera 11, Trieste, Italy

(C) EDP Sciences 
Signatures of ultrametricity can be found also in the dynamical behaviour of the system. An aging system may present a kind of dynamical ultrametricity [5]. In this case correlations between states at three long times $t_{1} \gg t_{2} \gg t_{3}$ should obey, asymptotically, the triangular relation:

$$
C\left(t_{1}, t_{3}\right)=\min \left\{C\left(t_{1}, t_{2}\right), C\left(t_{2}, t_{3}\right)\right\}
$$

It is difficult to test this relation in simulations due to strong preasymptotic time effects. An indirect test by constraining two real replicas to have one of the equilibrium values of the overlap was implemented in [9] for the three dimensional Edwards-Anderson spin glass. Another approach has been proposed in [10] by considering how dinamical ultrametricity should look like in a system subject to a weak external drive. When a system is subject to an external driving force, aging is eventually interrupted and stationary dynamics is attained after a characteristic time $\tau_{\epsilon}$, which depends on the strength of the drive, $\epsilon$. Interestingly, in the case when the three times are strongly separated, the relation (5) can be translated to the stationary dynamics:

$$
C\left(t_{1}-t_{3}\right) \equiv C\left(t_{1}-t_{2}+t_{2}-t_{3}\right)=\min \left\{C\left(t_{1}-t_{2}\right), C\left(t_{2}-t_{3}\right)\right\}
$$

In the preasymptotic region one expects that, approximately:

$$
C\left(t_{1}+t_{2}\right)=f\left[C\left(t_{1}\right), C\left(t_{2}\right)\right]
$$

This approach is interesting because it is conceptually simple and, in principle, can be tested straitforwardly in computer simulations. In fact, in ref. [10], the method was applied to a driven Edwards-Anderson spin glass in three dimensions, with inconclusive results. In this letter we perform a similar study on the four dimensional EA spin glass with asymmetric couplings. Our results are very different from those obtained in three dimensions, and the presence of dynamical ultrametricity is clearly established. Our results suggest that, in order to detect ultrametricity in finite dimensional models, the drive must in fact be very small and consequently very long time scales need to be reached. This is at variance with the observed behaviour in mean field models.

Four dimensional Edwards-Anderson spin glass. - The model is defined by the Hamiltonian:

$$
H=-\sum_{<i, j>}^{N} s_{i} J_{i j} s_{j},
$$

where $\left\{s_{i}= \pm 1, i=1 \ldots N\right\}$ are $N$ Ising spins and $\langle i, j\rangle$ denotes a sum over nearest neighbors. A drive can be applied to the system by adding a non Hamiltonian contribution to the energy. In a spin glass this can be accomplished, for example, by adding a non symmetric part to the copulings matrix. In this case the couplings $J_{i j}$ can be chosen as a weighted sum of a symmetric and a completely asymmetric part [11]:

$$
J_{i j}=\frac{1}{\sqrt{1-2 \epsilon+2 \epsilon^{2}}}\left[(1-\epsilon) J_{i j}^{(S)}+\epsilon J_{i j}^{(N S)}\right] .
$$

The symmetric part of the interaction is given by $J_{i j}^{(S)}=J_{j i}^{(S)}= \pm 1$ with probability 0.5 . The non-symmetric part $J_{i j}^{(N S)}$ is chosen independently of $J_{j i}^{(N S)}$. Finally, $\epsilon$ measures the strength of the non-symmetric part (the drive). 


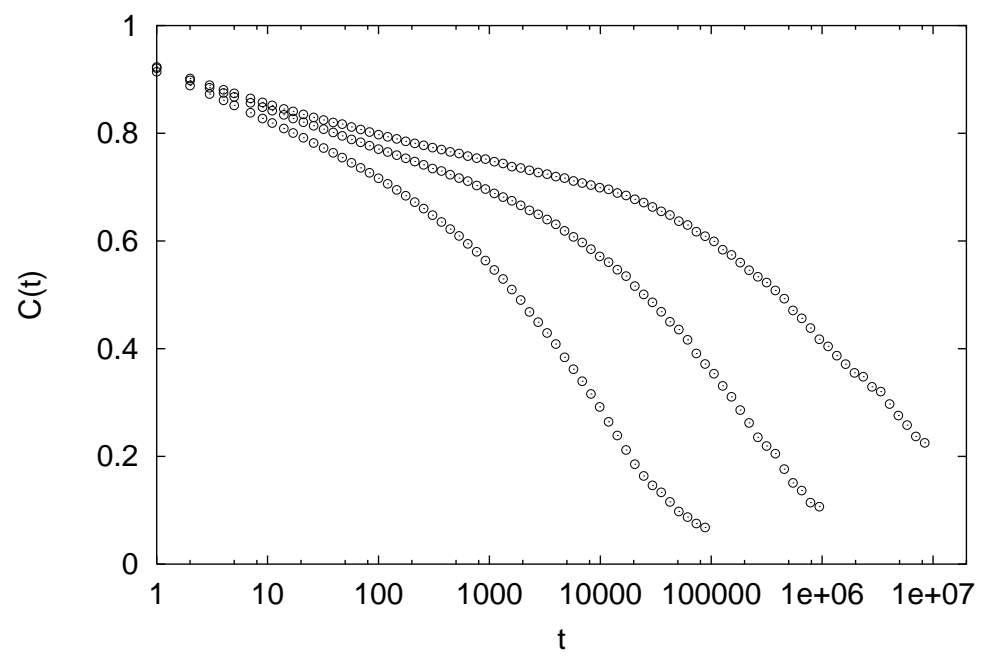

Fig. 1 - Stationary autocorrelations for three values of the drive, from top to bottom $\epsilon=0.1,0.15$ and 0.2 .

We let the system evolve until it reaches the stationary regime and then measured autocorrelations:

$$
C\left(t_{1}-t_{2}\right)=\frac{1}{N} \sum_{i=1}^{N} s_{i}\left(t_{1}\right) s_{i}\left(t_{2}\right)
$$

The measures were done on a system of linear size $L=10$ at temperature $T=1$, approximately $0.5 T_{c}$, for three different values of the driving force $\epsilon=0.1,0.15$ and 0.2 .

In the limit $\epsilon \rightarrow 0$ the correlations develop a plateau at a value $C \approx q_{E A}$. The development of the plateau can be observed only in the limit of very small drive, when the time scales for structural relaxation become very large, as can be seen in fig. 1. This growth of relaxation time when $\epsilon \rightarrow 0$ is equivalent to what happens in an aging system when the waiting time $t_{w} \rightarrow \infty$.

Dynamical ultrametricity implies the existence of a hierarchy of time scales. In particular this implies that the correlation will stay at nearly constant values for successively longer times as the system relaxes. This should manifest ideally in the development of a series of plateaus in the correlation as discussed in [10]. In such a scenario it is clear that a scaling of the autocorrelations assuming a single relevant time scale, $\tau(\epsilon)$, should fail. This simple scaling means that

$$
C_{\epsilon}(t) \propto f\left(\frac{t}{\tau(\epsilon)}\right)
$$

In fig. 2 we show that in fact this does not work in the four dimensional spin glass. We tried to scale the times with the time at which the correlations relaxed at $\mathrm{C}=0.5$, this value being well below the plateau at $q_{E A} \approx 0.7$. The three curves corresponding to the three different drivings meet only at $\mathrm{C}=0.5$, by construction, and cross each other at that point. This behaviour is similar to what happens in systems with full replica symmetry breaking, like the Sherrington-Kirkpatrick model or the Hofield neural network model [2, 12], which are ultrametric. A stronger evidence of dynamical ultrametricity is the presence of a logarithmic 


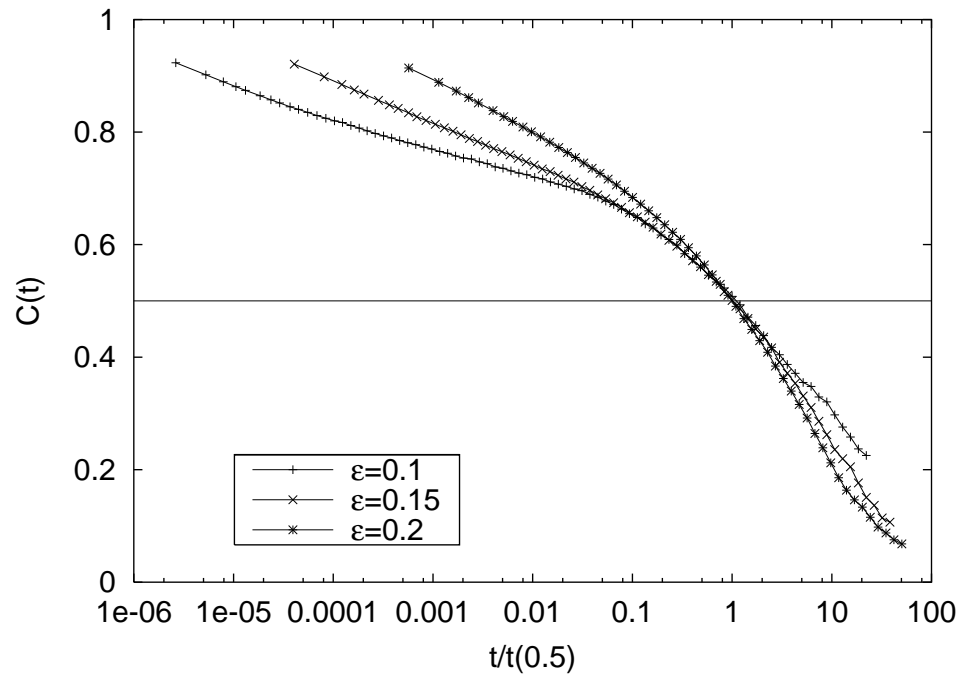

Fig. 2 - Simple scaling of the stationary autocorrelations for the three values of the driving force $\epsilon=01,0.15$ and 0.2 . Times are rescaled by the values at which the respective correlations decay to 0.5 .

scaling of the form

$$
C_{\epsilon}(t) \propto f\left(\frac{\ln (t)}{\ln (\tau(\epsilon))}\right)
$$

A scaling of this type is shown in fig. 3. It is much better than the previous simple scaling and is excellent for the two smaller drivings. This is an indication that the relevant results can only be reached approaching effectively the asymptotic regime of $\epsilon \rightarrow 0$.

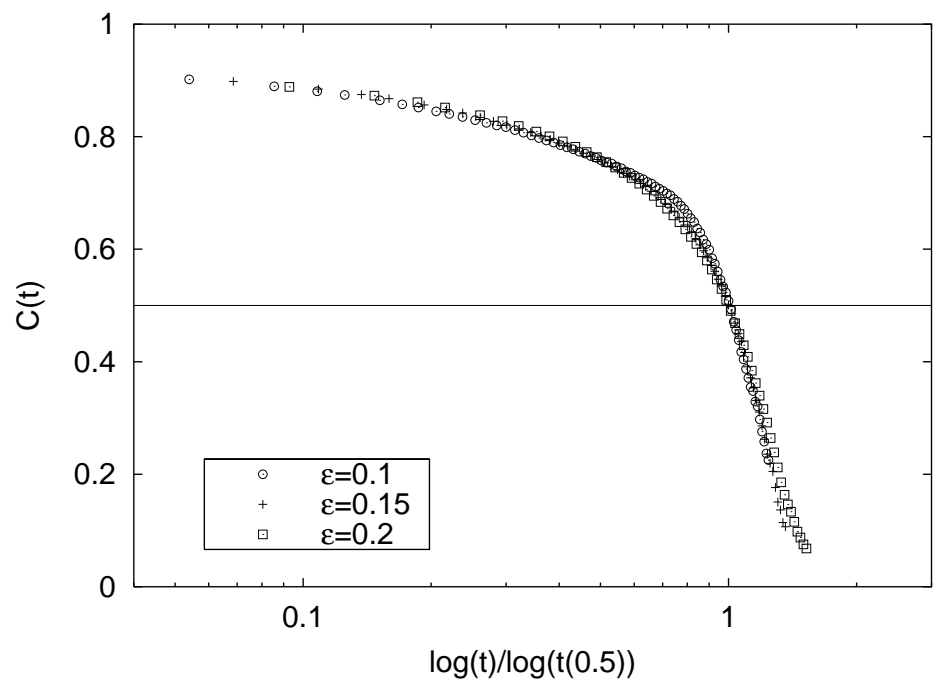

Fig. 3 - Logarithmic scaling of the stationary autocorrelations for the three values of the driving force $\epsilon=01,0.15$ and 0.2 . 
It is easy to show that a scaling of the form (12), in the presence of a small driving force, implies dynamic ultrametricity [10]. In fact, eq.(12) implies

$$
t\left(C_{\epsilon}\right) \propto(\tau(\epsilon))^{j\left(C_{\epsilon}\right)}
$$

where $j\left(C_{\epsilon}\right)$ is the inverse of $f$, a positive decreasing function of the correlation. Considering two values of the correlations such that $C_{1}<C_{2}<q_{E A}$ one finds that:

$$
\frac{t\left(C_{1}\right)}{t\left(C_{2}\right)} \propto(\tau(\epsilon))^{j\left(C_{1}\right)-j\left(C_{2}\right)} \stackrel{\epsilon \rightarrow 0}{\longrightarrow} \infty
$$

When, as in this case, the two times become assymptotically infinitely separated, this together with eq.(6) implies that $C\left(t\left(C_{1}\right)\right)=\min \left\{C\left(t\left(C_{1}\right)\right), C\left(t\left(C_{2}\right)\right)\right\}$, which is a realization of the ultrametric relation eq.(5) as applied to the stationary dynamics at finite $\epsilon$.

We have performed an analysis similar to the previous one to the four dimensional EA spin glass with gaussian couplings and without drive, i.e. at $\epsilon=0$. The resulting aging dynamics in that case was studied in [13]. The two time autocorrelation was found to scale as:

$$
C\left(t_{1}, t_{2}\right)=\left(q_{E A}+a\left(t_{1}-t_{2}\right)^{-x}\right) \frac{f\left(\frac{t_{1}-t_{2}}{t_{2}}\right)}{f(0)}
$$

with the scaling fuction:

$$
f(z)= \begin{cases}\text { constant } & \text { for } z \rightarrow 0 \\ z^{-\lambda(T)} & \text { for } z \rightarrow \infty\end{cases}
$$

This scaling gives an autocorrelation in the aging regime that is not ultrametric, at variance with our results in the driven system. The difficulty in observing ultrametricity during aging dynamics was already noted in [9]. Nevertheless, Eq.(15) is not the only scaling form which correctly fits the data for the autocorrelation functions in the aging regime. Also ultrametric scaling forms can be used with equivalent results [14]. Clearly the definitive scaling during aging is still not known.

As a last test, we have calculated the function $f\left(C_{1}, C_{2}\right)$ of eq.(何. As we are proving the preasymptotic regime, this function can give a flavour of the tendency of the triangular relation to become ultrametric or not. In this driven dynamics scenario, ultrametricity means that, as $\epsilon \rightarrow 0, f\left(C_{1}, C_{2}\right) \rightarrow \min \left(C_{1}, C_{2}\right)$. In other words, the curves of constant $f$ projected in the $\left(C_{1}, C_{2}\right)$ plane should tend to have right angles 10. In fig. 1 we show our results for the $4 \mathrm{D}$ EA spin glass.

Each group of three curves correspond to a fixed value of $f$ and the three values of the drive parameter studied. In each of these groups upper lines correspond to $\epsilon=0.2$, middle lines to $\epsilon=0.15$ and lower lines to $\epsilon=0.1$. The last group shows only two lines corresponding to $\epsilon=0.2$ and $\epsilon=0.15$ because of the lack of data for the smaller $\epsilon=0.1$ in this region of very small correlations. This figure should be compared to the corresponding one for the three dimensional Edwards-Anderson model of reference [10]. In the four dimensional case the separation of the lines becomes stronger on longer times. Also, for the lower values of $f$ a well defined tendency towards right angles is seen upon decreasing $\epsilon$, i.e. correlations are flowing in such a way as to satisfy dynamical ultrametricity as the drive $\epsilon \rightarrow 0$ at long times.

In the framework of the rheological approach to glassy dynamics, we have presented strong evidence of an ultrametric structure underlying long time correlations in the four dimensional +-J Edwards-Anderson spin glass. In comparing our results with the corresponding ones on the three dimensional model of [10] some comments are in order: first, we tried to get closer 


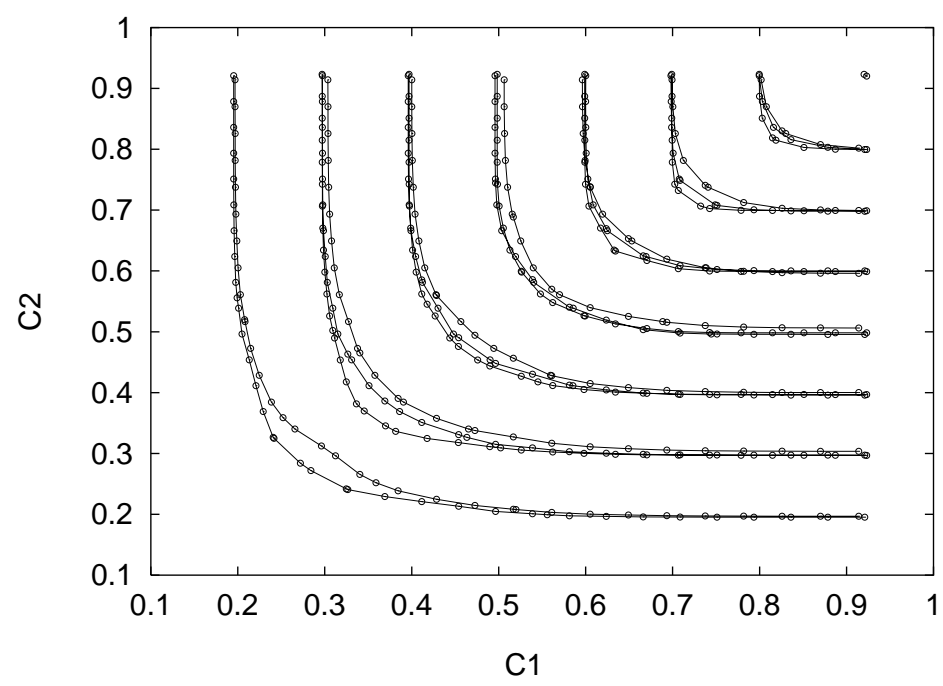

Fig. 4 - Projections on the plane $\left(C_{1}, C_{2}\right)$ of some constant values of the function $f$ defining the triangular relation eq.( curves correspond, from top to bottom, to $\epsilon=0.2,0.15$ and 0.1 (see text)

to the asymptotic regime $\epsilon \rightarrow 0$ in which analytical results are valid. Note that, in fact, the logarithmic scaling of fig. 3 is very good only for $\epsilon<0.2$. Of course, the price to pay is a strong growth of the relaxation time on decreasing $\epsilon$, as can be seen in fig. 1. Also, in mean field models relaxation is faster and relevant results can be seen already in the preasymptotic regime, i.e. for not too small values of $\epsilon$. In three dimensions relaxation is still slower than in four dimensions because of the larger value of the dinamical exponent $z$. To have an idea of the time scales involved note that $z\left(T_{c}\right)=4$ in the mean field model, $z\left(T_{c}\right)=5.26$ in four dimensions and $z\left(T_{c}\right)=6.67$ in three dimensions. The difference is roughly $25 \%$ between 3 and 4 dimensions and $30 \%$ between 4 dimensions and mean field. The relaxation times in three dimensions grow with respect to four dimensions as:

$$
\tau_{3 d}\left(T / T_{c}\right) \approx \tau_{4 d}\left(T / T_{c}\right)^{z_{3 d} / z_{4 d}}
$$

While at present it is a very hard problem, the three dimensional case should be accesible in the near future.

Once more, the four dimensional spin glass turns out to be a very valuable tool for addressing the question as to what extent some characteristics of the rich phenomenology of mean field spin glasses are present in finite dimensions below the upper critical one. Finally, probably results similar to ours can be observed in three dimensions on much longer time scales than those studied up to now.

$$
* * *
$$

I wish to thank the Abdus Salam ICTP for warm hospitality, where this work was finished. I want to thank also J. Kurchan, G. Parisi, F. Ricci-Tersenghi and A. Crisanti for useful comments. This work was supported in part by Conselho Nacional de Desenvolvimento Cientifico e Tecnologico (CNPq), Brazil. 


\section{REFERENCES}

[1] R. Rammal, G. Toulouse and M. Virasoro, Rev. Mod. Phys., 58 (1986) 765.

[2] M. Mézard, G. Parisi and M. Virasoro, Spin Glass Theory and Beyond (World Scientific, Singapore) 1987.

[3] A. K. Hartmann, Europhys. Lett., 44 (1998) 249.

[4] A. Cacciuto, E. Marinari and G. Parisi, J. Phys. A, 30 (1997) L263.

[5] L. F. Cugliandolo and J. Kurchan, J. Phys. A, 27 (1994) 5749.

[6] L. F. Cugliandolo and J. Kurchan, Philos. Mag. B, 71 (1995) 501.

[7] S. Franz and M. MÉzArd, Europhys. Lett., 26 (1994) 209.

[8] S. Franz and M. MÉzard, Physica A, 210 (1994) 48.

[9] F. Ricci-Tersenghi and S. Franz, Phys. Rev. E, 61 (2000) 1121.

[10] L. Berthier, J. L. Barrat and J. Kurchan, Phys. Rev. E, 63 (2001) 16105.

[11] E. Marinari and D. A. Stariolo, J. Phys. A, 31 (1998) 5021.

[12] M. Montemurro, F. A. Tamarit, S. A. Cannas and D. A. Stariolo, Phys. Rev. E, 62 (2000) 5721.

[13] G. Parisi, F. Ricci-Tersenghi and J. J. Ruiz-Lorenzo, J. Phys. A, 29 (1996) 7943.

[14] F. RiCCI-TERSENGHi, private communication. 